\title{
Congenital Heart Disease - A Review of the Treatment Methods
}

\section{Cynthia Wong* \\ Monash University, Australia \\ *Corresponding author: Cynthia Wong, Monash University, Australia}

\begin{abstract}
This paper seeks to provide a review of the existing treatment methods for Congenital Heart Disease. The paper will focus on the existing treatment methods for CHD, such as cardiac catheterization, patent ductus arteriosus (PDA), balloon valvulop, percutaneous mitral valve repair, hybrid intraoperative pulmonary artery stenting, catheter ablation, atrial septal defect (ASD), and transcatheter pulmonary valve therapy. It will also analyze coarctation of the aorta, balloon atrial septostomy, tricuspid atresia repair, hypoplastic left heart repair and finally, aortic stenosis. Despite the fear and belief among individuals that $\mathrm{CHD}$ is a lifelong disease that can lead to death once discovered in a patient, adopting the right treatment procedure can promote healthy living.
\end{abstract}

\section{Keywords}

Congenital heart disease, Existing treatments, Patients

\section{Introduction}

\section{Issues involved with CHD}

Children or adults living with CHD may develop complications during their lifetime [1]. These complications include developmental problems where the patients may notice some delays in different developmental processes. For instance, children might take a long time to work or talk properly. Some might find trouble in school due to poor oxygen supply to the brain, which affects the brain's development [1]. Learning difficulties include the following: Poor planning abilities, problems understanding language, impaired memory, and poor impulse, which may cause control individuals to act without thinking about the consequences of their actions [1].

\section{Endocarditis}

Endocarditis is a rare infection in the valves and the heart's lining, which, if not detected and consequently treated earlier, can cause life-threatening damages [2]. People living with CHD tend to have a higher risk of being affected with endocarditis [2]. This condition starts in another part of the body asan infection and spread through the blood into the heart.

\section{Pulmonary hypertension}

In certain forms of congenital heart diseases, the arteries' blood pressure connecting a person's heart and parts of the lungs becomes much higher than the normal level. A rapid heartbeat characterizes pulmonary hypertension, difficult breathing, feeling faintness, dizziness, and episodes of chest pains.

\section{Heart rhythm problems}

Infants and adults living with CHD risk developing different heart rhythm problems [2]. When a person is at rest without engaging in any physical activity, the heart should have between 60 to 100 rates every minute, which is the normal rate. Having heart rhythm problems might cause the heart to beat too slow or beat too fast. Under circumstances where a person experiences heartbeat at a slow rate, regulating the heart needs a pacemaker, and if it beats too fast, it may require medication [2]. In children, though rarely, an implant of a cardioverter-defibrillator is done to deliver a controlled electric shock to the heart and stop the rhythm issues [2]. Atrial fibrillation and atrial flutter are the two fast rhythms originating from the heart and advance with age. 


\section{Sudden cardiac death}

Despite the existence of a lower risk of cardiac death among individuals living with CHD, those with high risk have ventricular arrhythmias [3]. However, medics usually fit implantable cardioverter-defibrillator to sustain them.

\section{Heart failure}

Heart failure is a result of the heart's inability topu$\mathrm{mp}$ blood insufficient supply, thus failing to meet the blood level as per the body's demands. People with severe $\mathrm{CHD}$ are more prone to develop heart failure if they do not get proper treatment [2].

\section{Blood clots}

The probability of blood clots formingthat interfere with the normal heart's functioning is relatively high in patients having CHD history [4]. This can cause stroke or pulmonary embolism. When there is blockage of blood supply hindering adequate supply to the brain, this is called stroke [4]. Pulmonary embolism entails the event where blood supply has been blocked from reaching the lungs.

\section{Key Terminologies}

Echocardiogram- is a heart scan.

Pacemaker- is a small device used to control heartbeat and is placed beneath the skin close to the heart.

Cardioverter-Defibrillator- is used to keep track of the heart's rhythm and detect irregular heartbeats. The defibrillator can deliver an electric shock to stimulate the heart once it notices an abnormal rhythm.

Arrhythmia- is a rare condition where a person's heartbeats in an abnormal rhythm.

Atrial Septal Defect (ASD)- is a severe defect of the heart defect characterized by the existence of a hole in the walls dividing the upper chamber (right atria and left atria).

Ventricular Septal Defect (VSD)- is a condition where a hole exists in the septum, a hole separating the ventricles, such as the lower pumping chamber.

Atrioventricular Septal Defect- is a defect that affects the heart's right and left side's valves. The valves might not have formed correctly.

Coarctation of the aorta- is a condition where the aorta becomes narrower than the usual size. The left ventricle muscles are strained to push blood from the heart to the whole body.

Pulmonary Atresia- is when the valve controlling the blood flow from one's heart to their lungs does not function.

Pulmonary stenosis- is a condition that involves narrowing of a person's pulmonary valve and, in some instances, it extends to the pulmonary artery.

Tricuspid Atresia- is a condition where the valve controlling the flow of blood from the heart's right upper chamber to the right lower chamber malfunctions.

Ventricular hypertrophy- is a condition where the right ventricle is thicker than normal.

Truncus Arteriosus- is a rare heart defect where one vessel directs blood out of the heart instead of the aorta and the pulmonary artery.

Catheter-is a medical device inserted into a patient's body during surgical procedures.

\section{Background Information}

A congenital heart defect (CHD) refers to the heart vessels' structural defects. It is mostlynoticed at birth and it affects cardiac functions. CHDs are among the most typical defects observed in newborns, affecting 1 in 145 live births [5]. Research by Gilboa, et al. [6] indicates that Ventricular septal defect (VSD) exists among the most common CHD conditions. It occurs in 3.5 per 1000 live births [6]. Small VSDs, small Patent Ductus Arteriosus (PDA), as well as the Atrial Septal Defects (ASDs) are considered the least severe among $\mathrm{CHDs}$ and rarely cause problems to the patient since they close naturally [7]. Severe ones include Tetralogy of Fallot, instances of double outlet right ventricle, the Persistent Truncus Arteriosus, Atrioventricular septal defects, transposition of the great arteries, and large VSDs [8].

The most common type of congenital disabilities is congenital heart defects; however, most infants born with this condition will live to adulthood and have ordinary healthy lives [9]. One, out of every 120 babies, is born with heart disease. The disease usually occurs during early pregnancy when the baby's heart is forming. However, not all cases are serious, with one out of every four babies developing critical CHD [9]. A study in 2016 showed that about one million children and approximately 1.4 million adults live with CHD [10].

A cardioverter-defibrillator is used to keep track of the heart's rhythm and detect irregular heartbeats [11]. The defibrillator can deliver an electric shock to stimulate the heart once it notices an abnormal rhythm. The defibrillator, unlike the pacemaker, is not always active unless it senses an irregularity as compared to the pacemaker, which is always guiding the heart to maintain a steady heartbeat. Defibrillators act as resuscitation of the heart.

\section{Cardiac Catheterization Procedure}

When using a Cardiac catheterization procedure, a doctor, using X-ray or ultrasound imaging, inserts a catheter into a noticeable blood vessel either in the arms, neck, or groin and guides it to a patient's heart. Small specialized tools are then inserted using the catheter to correct the heart defect [12]. A good example is im- 
plantable cardiac closure devices which effectively plug holes in a septum. As time progresses, tissues grow over the implants placed on the walls that separate the heart's chambers [12]. These devices could be made from polyester, wire mesh, or both. The penetrating instrument's angle must be considered to transmit force towards the same direction with considerable force across a patient's vascular occlusion. It must be on a direct line or a straight line with the occlusion cap [13]. Technologies such as a guide catheter fitted with a co-axial hydrophilic catheter mounted with Mullins sheaths or Cook Medical with long sheaths is useful in these situations. With advancements in technology, new catheters are relatively short, and therefore do not require any modification. They can connect to the Y-shaped adapters if they exist in their original form, whereby glide catheters could easily be developed. Modification may still be necessary when dealing with taller patients. Sheaths such as the Oscor Destino Twist and Agilis NXT are steerable and available to doctors in medical facilities. They can shorten procedure time, especially when engaging in lesions that are in acute angles. Using the existing traditional methods such as the femoral artery may not be useful because of the vessels' acute angles [13].

The distal end is left uncoated to assist in the lesion's penetration, while the rest remains hydrophilic to ease advancement after crossing the lesion. For better support and force application, the wire's core is made of stainless steel. Occasionally, the guidewire will only move a short distance despite the sufficient support provided by guide catheters and the use of sheaths. In such instances, a micro-catheter is used to advance over the guidewire serving several objectives. First, sufficient support is achieved for the guidewire when the micro-catheter is advanced, allowing snaring from the other end [14]. Secondly, access to vessels that would otherwise be challenging is made possible with a microcatheter, available with angles at the tip. Finally, a guidewire can be exchanged for one with adequate support after ascertaining that the micro-catheter has become distal enough to sustain the procedure. While removing or withdrawing the guidewire, using the same wire which is shaped might facilitate re-entry to the lumen [14]. Several devices can help operators in such instances. Qureshi, et al. [14] note that it is an effective method in the restoration of vessel patency and the safest way.

\section{Transcatheter Pulmonary Valve Therapy}

Transcatheter pulmonary valve therapy or replacement is a treatment that can be used on individuals having narrow or leaky pulmonary valves without resorting to open-heart surgery [15]. When performing this procedure, a catheter holding an artificial valve is inserted through a vein [16]. It is then fitted in a patient's pulmonary artery. After which, a balloon is inflated, expanding the artificial valve into place. This treatment enables blood to flow well between the lungs and the right ventricle [17].

For individuals with pulmonary or aortic valve stenosis, a treatment known as balloon valvuloplasty is used to separate the thin wall leaflets, therefore, regulating blood flow [18]. A cardiologist opens the valve's leaflets, which are stiffened and narrowed using a small balloon. The doctor inserts a catheter into a vein with the aid of fluoroscopy, a continuous X-ray, providing a real-time image of the heart. The balloon placed at the tip is then expanded to split the leaflets. Both the balloon and the catheter are removed carefully through the same vein. Percutaneous mitral valve repair is a treatment procedure used to repair a leaky mitral valve [19]. A specialist fits a catheter into the vein and advances it to a patient's heart, specifically the left atrium or upper chamber [19]. Tiny polyester is inserted and placed above the leaky valve using the catheter to grasp the leaflets easily. This treatment enables the flaps to close well, facilitating blood flow in the heart.

In recent years, performing pulmonary valve replacement has provided treatment options for various forms of CHD. An excellent example is the truncus arteriosus, previously considered as an untreatable condition [17]. After a thorough physical examination and reviewing the lab imaging investigation, the procedure is performed, including computed tomography and echocardiography (CT/CMR). The process takes place in the catheterization laboratory, where the patient is under endotracheal anaesthesia [20]. It can be performed through the internal jugular vein and, in some instances, the femoral vein.

After gaining access, the physician injects intravenous heparin into the patient's system, which is used to attain activated clotting time (ACT) of between > 200s and 250s [20]. Standard right heart catheterization is conducted to access the pressure and gradient in the dysfunctional conduit. Angiography procedure then follows where a straight frontal and lateral with 200 cranial. After that, simultaneous selective coronary angiography together with non-compliant angioplasty balloon in a patient's right ventricular outflow tract conduit. To achieve a balance in placing the valve without compressing the coronary arteries, a distance of $10 \mathrm{~mm}$ is sufficient from the inflated balloon's end to the left coronary artery's beginning point. To ensure that the delivery is possible, an angiographic assessment of the heart's right ventricular outflow tract as well as the right ventricle's anatomy and size is essential.

\section{Catheter Ablation}

Catheter ablation is a treatment used on individuals with heart rhythm disorder, such as atrial fibrillation. Doctors completely remove tissues that cause abnormal rhythm in the atria. Destroying the abnormal electrical pathways using extremely high heat, radiofrequency 
energy, or extremely low temperature forms a scar that cannot conduct electricity. This procedure is performed under sedation while the catheter is guided by $\mathrm{X}$-ray imaging [19]. The radiofrequency electrical current is delivered through the catheter. As technology advances, it facilitates development diagnosis, early intervention, and treatment, and as a result, $90 \%$ of these patients reach adulthood [21].

When performing this procedure, the patient is given a sedative to help them relax. The skin on the groin, neck, or arm will be cleaned and made numb with an anaesthetic where the physician will make a small cut [22]. A catheter will then be inserted into the blood vessel, and with the use of X-ray imaging, the catheter will be advanced to the heart. In other cases, more than one catheter is used or needed per patient. As soon as the catheter is in place, physicians will connect small electrodes on the heart, which allows them to view the heart and locate which part of the heart has a problem [22]. As soon as the area is located, the catheter is used to deliver electrical energy, which leaves a small scar causing the rhythm problem to cease.

\section{Patent Ductus Arteriosus (PDA)}

Ductus arteriosus is an important element in foetal circulation, enabling the transfer of signals between a patient's aorta and the pulmonary artery. Usually, it closes two days after birth [23]. Patent ductus arteriosis (PDA) is officially diagnosed after 72 hours when it fails to close. PDA ends up redistributing the systemic blood flow by increasing the pulmonary blood flow, ventricular volume, and the left atrial. About $55-70 \%$ of pre-mature births or infants weighing less than $1000 \mathrm{~g}$ show haemodynamic symptoms from PDA [23]. Children showing these symptoms require urgent surgical or medical intervention. The procedure can primarily be performed under echocardiographic guidance [24]. In the neonatal unit, fluoroscopy supervision is advised while in the cardiac catheterization lab. Initially, both the arterial and femoral access needed to be acquired to be utilized as an important guide for the distal end of the ductus. A recent technological invention only needs access to the femoral venous and the esophageal temperature probe positioning [25].

The ductus is engaged into the descending aorta by travelling through a catheter into the patient's pulmonary artery. First, the PDA morphology is described by performing an angiogram to acquire its measurements. Coil embolization can be used occasionally on PDA cases with a pulmonary end diameter, which should be less than $2.5 \mathrm{~mm}$ [25]. These coils are made of spring wire covered in polyester fiber and are delivered by the use of a catheter. Occlusion occurs when a framework is created with wire and fabric that clots. When the occlusion device is loaded to the PDA, a guidewire is always used as an anchor. Several devices are used for PDA closure, such as; two-disk duct occluders and vascular plu- gs. The ductus diameter and the device should be the same [25]. The device is deployed when it is well placed in the ductus arteriosus with no protrusions on either side, residual shunting checked by echocardiography and a confirmation that there is no blockage to the descending aorta or branch pulmonary arteries. Another method involves making a small incision on the chest's left side. The operating physician then finds the PDA and either clips it off or divides and cuts the ductus arteriosus, otherwise known as ligation.

\section{Hybrid Intraoperative Pulmonary Artery Stenting}

Reconstructive pulmonary artery surgery in a repeat congenital heart surgery of pulmonary arteries can be challenging [26]. Although there are treatments such as survival patch angioplasty and percutaneous stent implantation that may suffice, the effective option with hybrid intraoperative stenting proves useful. Pediatric interventional cardiologists, in collaboration with pediatric cardiac surgeons, perform intraoperative stenting techniques and procedures. On most occasions, few patients require circulatory arrest. However, all the procedures are performed under hypothermic cardiopulmonary bypass. Before an operation, the area, size, and length of pulmonary artery stenosis are determined by either computerized tomographic pulmonary angiography or conventional angiography. The delivery balloon and the stent size are based on the angiographic images obtained. The reference points for this procedure include PA ostium and distal bifurcation [26]. Additionally, a physician using a Hegar capable of being introduced to the PA reconfirms the stenosed segment's diameter during the process.

\section{Atrial Septal Defect (ASD)}

All the patients undergoing ASD should go through standardized catheterization precautions, antibiotic prophylaxis anaesthesia, and heparin anticoagulation. Cardiac catheterization is done simultaneously to ensure proper positioning and elimination of the residual defect or valve obstruction. It is performed with a transesophageal echocardiogram (TEE). As it is with PDA, femoral venous percutaneous is the ideal access point. $A$ transesophageal echocardiogram then evaluates all the abnormalities associated with septal defects. Multiuse or Berman is then used to take the pressure and flow measurements. The wire serving as an anchor is placed in the left-upper pulmonary vein. Regular sizing of the balloon is necessary since the septal defects are not symmetrical and more often stretch [2]. Before an intervention, a stretched balloon and a well-sized ASD is normally $30 \%$ greater than the measured transesophageal echocardiogram [2]. Balloon sizing helps to determine hemodynamic properties in the heart and the chances of an individual tolerating closure. A surgical option is also available to repair ASD [27]. The septum is closed using stitches. However, a patch can also be used to cover the hole. 


\section{Coarctation of the Aorta}

The retrograde transfemoral approach is preferred in evaluating the femoral vein and artery. Special attention is necessary when accessing the artery to avoid splitting the femoral artery. This procedure ensures that a larger diameter vessel is attained, considering that larger stents need the sheath's corresponding size, ultimately increasing vascular compromise risk [28]. Patients undergoing coarctation of the aorta must also undergo a comprehensive right to left -heart catheterization and a thorough aorta angiogram [28]. Additional imaging can be done by rotational angiography. When preparing for stent placement, the physician places a firmer guidewire across the stenosis area. In addition to the procedure's precision, the cardiologist's experience plays an important role when choosing the stent. Ideally, the abdominal aorta diameter is more or equal to the diameter of the stent.

Moreover, the stent diameter's ratio to the narrowest part of the coarctation is less than 3.5 [28]. A balloon-in-balloon catheter is loaded once the stent is chosen [28]. When the stent is deployed partially, the inner balloon gives room for repositioning. When the treatment is successful, the residual gradient is less than 10 $\mathrm{mmHg}$ and a vessel caliber, above $80 \%$ of the adjacent aortic segment [28]. When choosing a surgical option to repair defects in the aorta, a cut is made between the ribs on the chest's left side. The ordinary way for repair involves dissecting the narrow section followed by its enlargement with a patch made of Gore-Tex, a synthetic material. The second way to repair this issue is known as the subclavian flap. A cut is made in the aorta, the narrow part. Then, a patch from the subclavian artery is used to broaden the narrow section. The third way to resolve this issue is by stitching the ends together after removing the aorta's narrow section. This technique is majorly used in older children. A fourth way to tackle this problem is by connecting the tube to the normal sections of the aorta. It is done on both sides of the narrow section to allow blood to flow through, bypassing the affected area.

\section{Balloon Atrial Septostomy}

With this procedure, access might sometimes be possible through the neonatal umbilical vein. However, the femoral approach is preferred when there are venous tortuosities. Guidance throughout this procedure is by transthoracic echocardiographic since infants are too small for a transesophageal echocardiogram. It is also important to note that the individual being treated should be on anti-coagulants with heparin in combination with antibiotic prophylaxis.

The medical procedure starts by moving the balloon through the restrictive septum from the right to the left atrium [29]. In cases like hypoplastic left heart syndrome, a thick atrial septum could be existing. A radiofre- quency wire perforation or a transseptal puncture needle may be used to deliver the balloon. Upon reaching the left atrium, the wire is inflated and bounces back to the right atrium for further deflation [29]. The procedure is done severally, facilitating the confirmation of the atrial shunt through echocardiography.

\section{Tricuspid Atresia Repair}

Tricuspid Atresia is an acromion form of CHD that occurs when the tricuspid valve, found between the heart's upper and lower chambers, is narrow, deformed, or missing [30]. Infants with this condition seem blue since blood cannot pick up oxygen in the heart. Immediately after birth, the infant is given a medicine known as prostaglandin $\mathrm{E}$. The medicine will help keep the patient's ductus arteriosus open, allowing blood to flow continuously to the lungs. This is a temporary measure to keep the child alive and stable; however, the child will need surgery eventually. Several shunts and surgeries will be needed to correct the defect. The aim is to allow blood to circulate freely from the body to the lungs [30]. The physician will need to replace the valve, repair the tricuspid valve and, or insert a shunt so that blood reaches the lungs.

\section{Hypoplastic Left Heart Repair}

It is a life-threatening defect caused by a poorly developed left side of the heart. If left unchecked or untreated, it caused death to most babies. Surgical treatment of this condition is done in specialized hospitals [31]. Firstly, a crucial and complicated surgery is done where a blood vessel is made from a patient's pulmonary artery or the aorta. Its work is to circulate the blood to the lungs and the body generally. The second procedure, known as the Fontan operation, is done when an infant is 4-6 months-old. This method redirects blood from the lower body to the lungs. In this approach, the Inferior Vena Cava (ICV) is joined to a patient's pulmonary artery through a 'conduit' tube. It is done after the IVC is detached from the heart [31]. On occasions, a small hole is made on the 'conduit' to connect the heart. As the lung adapts to the new blood flow level from the lower parts of the body, the small hole acts as a 'pop-off valve. The hole can be closed with cardiac catheterization. The third operation could be done a year after.

\section{Aortic Stenosis}

All the standard catheterization precautions should be adhered to while performing this procedure. In some cases, past the neonatal period, this technique could be performed when an individual is conscious. The best approach is retrograde transfemoral preferred in this procedure since access to the femoral artery and vein is easier [32]. A comprehensive right and left-heart catheterization and a thorough angiogram are done for all patients undergoing this procedure. Transvalvar gradient obtained by using a pullback with a catheter is the most 
important measurement [32]. A trans-atrial-septal approach or extra/additional arteries access point can also be used for measurement. The balloon's size should range from 80 to $90 \%$ of the actual annulus size to avoid a trivial aortic regurgitation risk. When the procedure is done, the gradient is measured to check for success. Ideally, results showing reduction of the gradient by half and lack of increase in aortic regurgitation is successful.

\section{Conclusion}

There still exists inadequate information about the exact causes of CHD. In many cases, the identification of a single factor causing the condition proves difficult. Genetics links and environmental factors or both are the most common causes [33]. Researchers have embarked on studies to identify the factors promoting CHD development [4]. For example, research notes that smoking among pregnant women, gestational maternal diabetes, and poor feeding and lifestyles during pregnancy are the main contributing factors. Low maternal folic acid ingestion levels among pregnant women, obesity, and exposure to various air pollutants such as pesticides increase the complication's risk. Pacemaker and Cardioverter defibrillator are common devices used to mitigate CHD effects and prevent death or more complications to patients [34]. The pacemaker is used to help the heartbeat more regularly for patients experiencing arrhythmia. Pacemakers are used by many patients suffering from CHD to ensure the rate of their heartbeat is kept in check [2]. Considering these approaches and the other potential ones that have not yet been proved effective, researchers need to examine the efficiency of the existing methods for treating CHD and facilitate the discovery of more approaches to improve affected persons' lives. Congenital heart disease is a serious condition that risks the lives of children and since it is difficult to identify the associated defects during pregnancy. Despite many individuals associating the health complication with early death, it can be managed if medics detect it during the initial stages.

\section{Conflicts of Interest}

On behalf of all authors, the corresponding author states that there is no conflict of interest.

\section{References}

1. Lantin-Hermoso MR, Berger S, Bhatt AB, Richerson JE, Morrow R, et al. (2017) The care of children with congenital heart disease in their primary medical home. Pediatrics 140: e20172607.

2. Zaidi S, Brueckner M (2017) Genetics and genomics of congenital heart disease. Circ Res 120: 923-940.

3. Katritsis DG, Gersh BJ, Camm AJ (2016) A clinical perspective on sudden cardiac death. Arrhythm Electrophysiol Rev 5: 177-182.

4. Hinton RB, Ware SM (2017) Heart failure in pediatric patients with congenital heart disease. Circulation Research 120: $978-994$.
5. Liu Y, Chen S, Zühlke L, Black GC, Choy M, et al. (2019) Global birth prevalence of congenital heart defects 19702017: Updated systematic review and meta-analysis of 260 studies. Int J Epidemiol 48: 455-463.

6. Gilboa SM, Salemi JL, Nembhard WN, Fixler DE, Correa A (2010) Mortality resulting from congenital heart disease among children and adults in the United States, 1999 to 2006. Circulation 122: 2254-2263.

7. Meyer M, Hreinsdottir A, Häcker A, Brudy L, Oberhoffer R, et al. (2018) Web-based motor intervention to increase health-related physical fitness in children with congenital heart disease: A study protocol. Front Pediatr 6: 224.

8. Martins P, Castela E (2008) Transposition of the great arteries. Orphanet Journal of Rare Diseases 3: 27.

9. Pierpont ME, Brueckner M, Chung WK, Garg V, Lacro RV, et al. (2018) Genetic basis for congenital heart disease: Revisited: A scientific statement from the American Heart Association. Circulation 138: e653-e711.

10. Thakkar A, Fuentes-Rojas S, Karanja E, Ebunlomo E, Millette A, et al. (2018) Building an adult congenital heart program: Critical components and important allies. Curr Cardiol Rep 20: 134.

11. Guerra F, Palmisano P, DellEra G, Ziacchi M, Ammendola E, et al. (2016) Implantable cardioverter-defibrillator programming and electrical storm: Results of the OBSERVational registry on long-term outcome of ICD patients (OBSERVO-ICD). Heart Rhythm 13: 1987-1992.

12. Holst KA, Said SM, Nelson TJ, Cannon BC, Dearani JA (2017) Current interventional and surgical management of congenital heart disease: Specific focus on valvular disease and cardiac arrhythmias. Circ Res 120: 1027-1044.

13. Latson LA, Qureshi AM (2010) Techniques for transcatheter recanalization of completely occluded vessels and pathways in patients with congenital heart disease. Ann Pediatr Cardiol 3: 140-146.

14. Qureshi AM, Hill JA, Prieto LR, Arruda J, Morrison S, et al. (2013) Transcatheter recanalization of totally occluded proximal pulmonary arteries and major systemic veins in patients with congenital heart disease. Am J Cardiol 111: 412-417.

15. Sharma V (2019) Congenital heart defects. Insights in Biomedicine 4: 8.

16. Dori Y, Keller MS, Rome JJ, Gillespie MJ, Glatz AC, et al. (2016) Percutaneous lymphatic embolization of abnormal pulmonary lymphatic flow as treatment of plastic bronchitis in patients with congenital heart disease. Circulation 133: 1160-1170.

17. Ghawi H, Kenny D, Hijazi ZM (2012) Transcatheter pulmonary valve replacement. Cardiol Ther 1: 5 .

18. Sun R, Liu M, Lu L, Zheng Y, Zhang P (2015) Congenital heart disease: Causes, diagnosis, symptoms, and treatments. Cell Biochem Biophys 72: 857-860.

19. Budts W, Roos-Hesselink J, Rädle-Hurst T, Eicken A, McDonagh TA, et al. (2016) Treatment of heart failure in adult congenital heart disease: A position paper of the working group of grown-up congenital heart disease and the Heart Failure Association of the European Society of Cardiology. Eur Heart J 37: 1419-1427.

20. McElhinney DB, Hellenbrand WE, Zahn EM, Jones TK, Cheatham JP, et al. (2010) Short- and medium-term outcomes after transcatheter pulmonary valve placement in the expanded multicenter US melody valve trial. Circulation 122: $507-516$. 
21. Köhler F, Schierbaum C, Konertz W, Schneider M, Kern $\mathrm{H}$, et al. (2005) Partnership for the heart: German-Estonian health project for the treatment of congenital heart defects in Estonia. Health Policy 73: 151-159.

22. Calkins H, Hindricks G, Cappato R, Kim Y, Saad E, et al (2019) Corrigendum to: 2019 HRS/EHRA/APHRS/LAHRS expert consensus statement on catheter ablation of ventricular arrhythmias. Europace 21: 1144.

23. EL-Khuffash A, Weisz DE, McNamara PJ (2016) Reflections of the changes in patent ductus arteriosus management during the last 10 years. Arch Dis Child Fetal Neonatal Ed 101: F474-F478.

24. Gillam-Krakauer M, Reese J (2018) Diagnosis and management of patent ductus arteriosus. Neoreviews 19: e394e402.

25. Freud LR, Tworetzky W (2016) Fetal interventions for congenital heart disease. Curr Opin Pediatr 28: 156-162.

26. Chakraborty B, Hagler D, Burkhart HM, Dearani JA (2013) Intraoperative hybrid left pulmonary artery stenting. Ann Pediatr Cardiol 6: 43-45.

27. Behjati-Ardakani M, Golshan M, Akhavan-Karbasi S, Hosseini SM, Behjati-Ardakani MA, et al. (2016) The clinical course of patients with atrial septal defects. Iran J Pediatr 26: e4649.
28. Faraoni D, Zurakowski D, Vo D, Goobie SM, Yuki K, et al. (2016) Post-operative outcomes in children with and without congenital heart disease undergoing non-cardiac surgery. J Am Coll Cardiol 67: 793-801.

29. Gopalakrishnan A, Sasidharan B, Krishnamoorthy KM (2016) Balloon atrial septostomy in congenital heart disease. Circ J 80: 1050.

30. Minocha PK, Phoon C (2020) Tricuspid atresia. StatPearls.

31. Brun H, Bugge RAB, Suther LKR, Birkeland S, Kumar R, et al. (2018) Mixed reality holograms for heart surgery planning: First user experience in congenital heart disease. Eur Heart J Cardiovasc Imaging 20: 883-888.

32. DiLorenzo MP, Santo A, Rome JJ, Zhang H, Faerber JA, et al. (2019) Pulmonary vein stenosis: Outcomes in children with congenital heart disease and prematurity. Semin Thorac Cardiovasc Surg 31: 266-273.

33. Hajar R (2017) Risk factors for coronary artery disease: Historical perspectives. Heart Views 18: 109-114.

34. Sawyer DB, Vasan RS (2017) Encyclopedia of cardiovascular research and medicine. Elsevier. 